\title{
PHOTOLUMINESCENCE AND PHOTOLUMINESCENCE EXCITATION SPECTROSCOPY OF IN SITU Er-DOPED AND Er-IMPLANTED GaN FILMS GROWN BY HYDRIDE VAPOR PHASE EPITAXY
}

\author{
S. KIM,* X. LI,* J. J. COLEMAN, ${ }^{*}$ R. ZHANG,** D. M. HANSEN, ** T. F. KUECH,** \\ S. G. BISHOP* \\ *Microelectronics Laboratory, University of Illinois at Urbana-Champaign, Urbana, IL 61801 \\ **Department of Chemical Engineering, University of Wisconsin, Madison, WI 53706
}

\section{Cite this article as: MRS Internet J. Nitride Semicond. Res. 4S1, G11.4(1999)}

\section{ABSTRACT}

Photoluminescence (PL) and photoluminescence excitation (PLE) spectroscopy have been carried out at $6 \mathrm{~K}$ on the $1540 \mathrm{~nm}^{4} \mathrm{I}_{13 / 2} \rightarrow{ }^{4} \mathrm{I}_{15 / 2}$ emission of $\mathrm{Er}^{3+}$ in in situ Er-doped and Erimplanted GaN grown by hydride vapor phase epitaxy (HVPE). The PL and PLE of these two different Er-doped HVPE-grown GaN films are compared with Er-implanted GaN grown by metal organic chemical vapor deposition (MOCVD).

In the in situ Er-doped HVPE-grown GaN, the lineshape of the broad PLE absorption bands and the broad PL bands is similar to that in Er-doped glass. The PL spectra of this in situ Er-doped sample are independent of excitation wavelength, unlike the PL of the Er-implanted $\mathrm{GaN}$. These PL spectra are quite different from the site-selective PL spectra observed in the Erimplanted $\mathrm{GaN}$, indicating that the seven different $\mathrm{Er}^{3+}$ sites existing in the Er-implanted MOCVD-grown GaN are not observed in the in situ Er-doped HVPE-grown GaN. Four of the seven different $\mathrm{Er}^{3+}$ sites observed in the Er-implanted MOCVD-grown $\mathrm{GaN}$ annealed at $900{ }^{\circ} \mathrm{C}$ under a flow of $\mathrm{N}_{2}$ are present in the Er-implanted HVPE-grown $\mathrm{GaN}$ annealed at $800{ }^{\circ} \mathrm{C}$ in an $\mathrm{NH}_{3} / \mathrm{H}_{2}$ atmosphere.

\section{INTRODUCTION}

Photoluminescence (PL) and photoluminescence excitation (PLE) spectroscopy have been performed to study the different structural configurations (sites or centers) on which $\mathrm{Er}^{3+}$ ions are incorporated in Er-doped $\mathrm{GaN}$ and to investigate their excitation mechanisms [1-7]. Our previous study of site-selective PL and PLE spectroscopy in Er-implanted samples of GaN grown by metal organic chemical vapor deposition (MOCVD) that were annealed at $900{ }^{\circ} \mathrm{C}$ under a flow of $\mathrm{N}_{2}$ revealed the existence of seven different $\mathrm{Er}^{3+}$ sites [3]. Six of these $\mathrm{Er}^{3+} \mathrm{PL}$ sites are attributed to complexes of Er atoms with defects and impurities. Only one of the seven sites can be pumped by direct $4 f$ absorption; the concentrations of the other sites are too low to allow excitation by direct $4 f$ absorption. These results raise an obvious question concerning the generality or uniqueness of the $\mathrm{Er}^{3+}$ centers observed in $\mathrm{GaN}$. That is, are these multiple, selectively excited, discrete $\mathrm{Er}^{3+}$ sites or centers unique to Er-implanted MOCVD-grown GaN subjected to specific post-implantation annealing conditions, or are they also present in Er-doped $\mathrm{GaN}$ synthesized by other growth, doping and annealing procedures?

Site-selective PL and PLE spectroscopies used in our previous work are applied in this study to Er-implanted hydride vapor phase epitaxy (HVPE)-grown $\mathrm{GaN}$ annealed at $800{ }^{\circ} \mathrm{C}$ in an $\mathrm{NH}_{3} / \mathrm{H}_{2}$ atmosphere and in situ Er-doped HVPE-grown GaN [7] to see if any of the seven 
different $\mathrm{Er}^{3+}$ sites observed in Er-implanted MOCVD-grown GaN exist in these samples. The PLE and PL of these two different HVPE-grown samples are compared here first with the Erimplanted MOCVD-grown sample and then the differences and similarities among the siteselective PLE and PL spectra of these three different Er-doped GaN films are discussed in detail.

\section{EXPERIMENTAL PROCEDURE}

The GaN films were doped in-situ with Er in a horizontal HVPE reactor during growth. A peak Er concentration of $2 \times 10^{19}$ ions $/ \mathrm{cm}^{3}$ was achieved in this in situ Er-doped GaN at a thickness of $1000 \mathrm{~nm}$ [7]. The GaN films grown on sapphire by HVPE were implanted with a dosage of $2 \times 10^{14}$ ions $/ \mathrm{cm}^{2}$ at $300 \mathrm{keV}$. The peak concentration of Er is $5.3 \times 10^{19}$ ions $/ \mathrm{cm}^{3}$ at a depth of $33 \mathrm{~nm}$ [7]. These Er-implanted HVPE-grown GaN films were annealed in a conventional tube furnace at $800{ }^{\circ} \mathrm{C}$ for 30 minutes in a flowing $\mathrm{NH}_{3} / \mathrm{H}_{2}$. For comparison, the GaN films grown on sapphire by atmospheric pressure MOCVD were implanted with a dosage of $4 \times 10^{13}$ ions $/ \mathrm{cm}^{2}$ at $280 \mathrm{keV}$ [1-4]. The peak concentration of Er is $2 \times 10^{18}$ ions $/ \mathrm{cm}^{3}$. Post-implantation annealing was carried out in a conventional tube furnace at $900{ }^{\circ} \mathrm{C}$ for 30 minutes under a continuous flow of nitrogen gas.

6K PL spectroscopy was carried out on the three different Er-doped GaN samples. The PL spectra were excited by a variety of sources including a tunable titanium-doped sapphire laser, a HeNe laser, an Ar ion laser, a Xe lamp dispersed by a double grating monochromator, and a HeCd laser. The PLE spectra were obtained with a xenon lamp dispersed by a double grating monochromator or with a tunable titanium-doped sapphire laser. All of the PLE spectra were corrected for the spectral response of the tunable excitation systems. The luminescence was analyzed by a 1-m single grating monochromator and detected by a cooled Ge PIN detector. Samples were cooled to liquid helium temperature in a Janis Supervaritemp Cryostat.

\section{RESULTS AND DISCUSSION}

Figure 1 shows the PL spectra obtained at $6 \mathrm{~K}$ under excitation by $515 \mathrm{~nm}$ light ("greenpumped") from in situ Er-doped and Er-implanted GaN grown by HVPE. The PL spectrum taken for Er-implanted MOCVD-grown GaN under the same experimental conditions is also shown for comparison. All three PL spectra exhibit the 1540 $\mathrm{nm}$ band characteristic of the ${ }^{4} \mathrm{I}_{13 / 2} \rightarrow{ }^{4} \mathrm{I}_{15 / 2}$ transitions of $\mathrm{Er}^{3+}$ and broad background PL bands on which the Er-related PL bands are superimposed. In the PL of the in situ Er-doped GaN (Fig. 1a), the broad PL bands have different lineshapes and peak positions from those of the damage-induced broad-band PL observed in the Er-implanted MOCVD-grown GaN (Fig. 1c) [4]. Since these bands have not been observed in light).

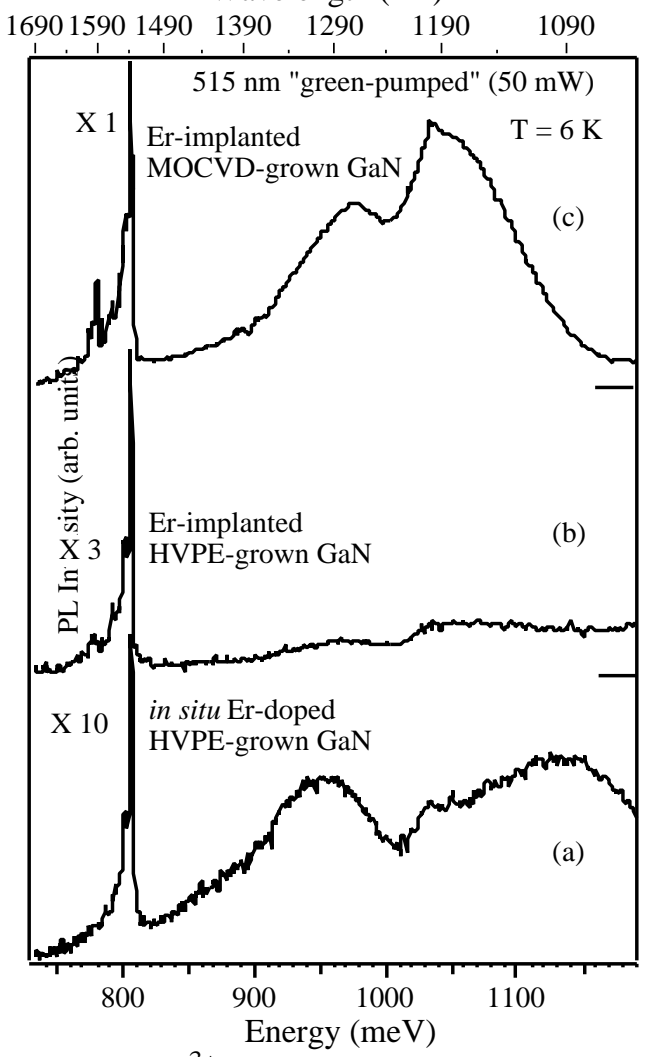

Fig. 1. The $\mathrm{Er}^{3+}$ PL spectra and the broad defect PL bands (pumped by $515 \mathrm{~nm}$ 
undoped HVPE-grown GaN, they are apparently induced by the in situ doping during growth. In the PL spectrum of the Er-implanted HVPE-grown GaN (Fig. 1b), the damage-induced broadband PL is barely observable.

Figure 2 displays the PLE spectra of the three different Er-doped samples obtained by detecting the integrated $\mathrm{Er}^{3+}$ PL intensity while scanning the wavelength of the xenon lampdouble monochromator excitation system. The PLE spectrum 2(a) of the in situ Er-doped sample exhibits relatively narrow absorption bands attributable to direct optical excitation of the intra $4 f$ shell transitions of $\mathrm{Er}^{3+}$, and an exponential absorption tail just below the $3.5 \mathrm{eV}$ band gap. The exponential energy dependence of the below-gap absorption tail is reminiscent of the Urbach absorption edge that characterizes the band edge of disordered materials such as chalcogenide glasses [8] or highly doped crystalline semiconductors [9]. This PLE spectrum does not show the broad, mid-gap defect- or impurity-related absorption bands that are observed in the PLE spectra 2(b) and 2(c) of the Er-implanted samples. The absorption peaks seen at 378, 400, 445, 487, 521, 543, 651, 796, and $970 \mathrm{~nm}$ in the PLE spectrum 2 (a) are attributed to ${ }^{4} \mathrm{I}_{15 / 2} \rightarrow{ }^{4} \mathrm{G}_{11 / 2},{ }^{4} \mathrm{I}_{15 / 2}$ $\rightarrow{ }^{4} \mathrm{H}_{9 / 2},{ }^{4} \mathrm{I}_{15 / 2} \rightarrow{ }^{4} \mathrm{~F}_{3 / 2,5 / 2},{ }^{4} \mathrm{I}_{15 / 2} \rightarrow{ }^{4} \mathrm{~F}_{7 / 2},{ }^{4} \mathrm{I}_{15 / 2} \rightarrow{ }^{4} \mathrm{H}_{11 / 2},{ }^{4} \mathrm{I}_{15 / 2} \rightarrow{ }^{4} \mathrm{~S}_{5 / 2},{ }^{4} \mathrm{I}_{15 / 2} \rightarrow{ }^{4} \mathrm{~F}_{9 / 2},{ }^{4} \mathrm{I}_{15 / 2} \rightarrow{ }^{4} \mathrm{I}_{9 / 2}$, and ${ }^{4} \mathrm{I}_{15 / 2} \rightarrow{ }^{4} \mathrm{I}_{11 / 2} \mathrm{Er}^{3+}$ intra 4 -shell transitions, respectively [5,6,10]; some of these PLE bands were reported in Refs. 5 and 6 to be observed in Er-implanted GaN and in situ Er-doped AlN grown by molecular-beam epitaxy (MBE). In contrast, the PLE spectra 2(b) and 2(c) obtained from the Er-implanted HVPE- and MOCVD-grown samples, respectively, show only the broad, defect- or impurity-related absorption bands that are not attributable to $\mathrm{Er}^{3+}$. These absorption bands are characteristic of the implanted films only, suggesting that some of the broad absorption bands in the PLE spectra 2(b) and 2(c) are associated with defects or defect-impurity complexes created during the implantation and annealing procedures $[1,3]$.

The high-resolution PLE spectra (Fig. 3) obtained in the 775-825 nm spectra range with

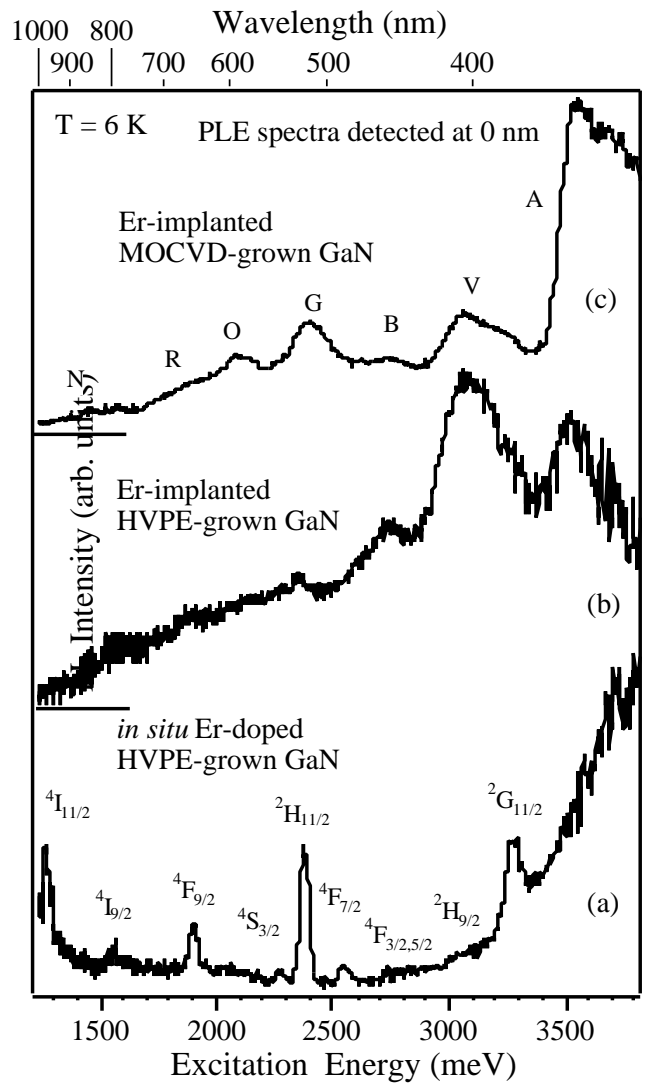

Fig. 2. The PLE spectra obtained by detecting the integrated $\mathrm{Er}^{3+} \mathrm{PL}$ intensity.

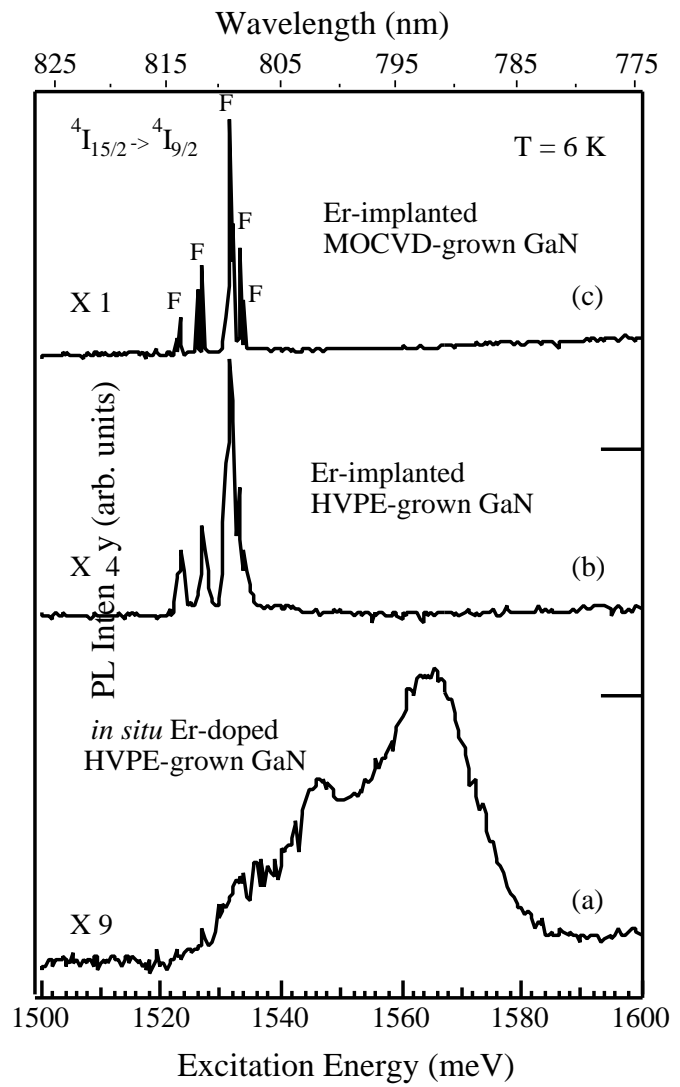

Fig. 3. The ${ }^{4} \mathrm{I}_{15 / 2} \rightarrow{ }^{4} \mathrm{I}_{9 / 2} 4 f$ shell Er ${ }^{3+}$ PLE spectra. 
the tunable Ti-doped sapphire laser exhibit PLE peaks assigned to direct ${ }^{4} \mathrm{I}_{15 / 2} \rightarrow{ }^{4} \mathrm{I}_{9 / 2} 4 f$ shell absorption. The absorption bands seen in the PLE spectrum (Fig. 3a) of the in situ Er-doped sample are much broader and their peak positions are shifted to the shorter wavelength region, compared to the Er-implanted samples. Note that the PLE spectrum of the MOCVD-grown GaN in Fig. 3c shows five pairs of sharp peaks, indicative of a single type of $\mathrm{Er}^{3+}$ center or site. The loss of the sharp structure in the PLE spectrum of the in-situ Er-doped GaN sample indicates that the Er dopants in this sample occupy sites located in disordered regions of the material. The disordered or "amorphous" character of the $\mathrm{Er}^{3+}$ centers' structural environments gives rise to site-to-site variations in the magnitude and symmetry of the crystal fields experienced by the $\mathrm{Er}^{3+}$ ions that are responsible for the spectral broadening of the $\mathrm{Er}^{3+} 4 f$ PLE bands [8,9]. Additional evidence for disorder in the structural environment of the Er dopants in the GaN doped during growth is provided by the broad Urbach absorption edge in the near-band edge PLE spectrum of Fig. 2a, which contrasts strongly with the relatively sharp band edge absorption features observed in the PLE spectra of the $\mathrm{Er}^{3+}$ emission in Er-implanted samples of GaN (see, for example, Fig. 2c). Furthermore, the $1540 \mathrm{~nm} \mathrm{Er}^{3+}$ PL bands from the in situ Er-doped sample shown in Figs. 1a, $5 \mathrm{a}$, and $7 \mathrm{a}$ all have a broadened line shape more characteristic of Er dopants in glasses [8], than the sharply structured $1540 \mathrm{~nm} \mathrm{Er}^{3+}$ PL bands of the Er-implanted GaN samples shown in Figs. 1b and c, $5 \mathrm{~b}$ and $\mathrm{c}, 6 \mathrm{a}$ and $\mathrm{b}$, and $7 \mathrm{~b}$ and $\mathrm{c}$.

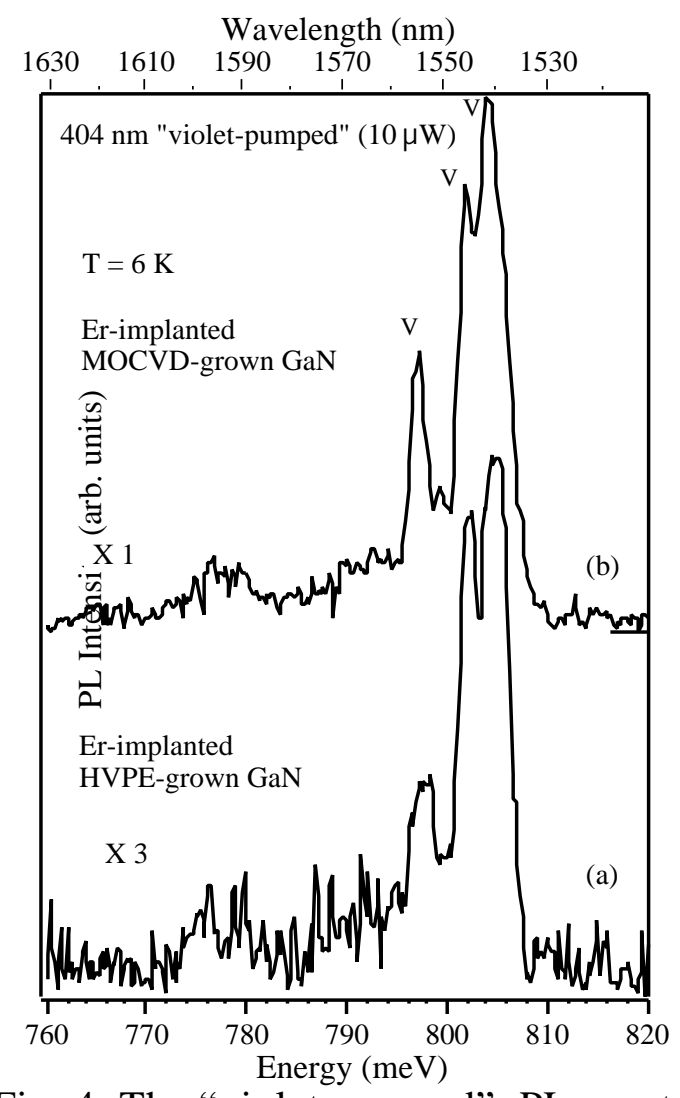

Fig. 4. The "violet-pumped" PL spectra as excited by violet light (404 $\mathrm{nm})$.

The PLE spectrum of the Er-implanted

HVPE-grown GaN in Fig. 3b exhibits sharp-structured peaks having the same peak positions as those in the Er-implanted MOCVD-grown GaN (Fig. 3c). A careful comparison between these two PLE absorption bands reveals that the PLE peaks in the HVPE-grown one are broader, compared to the MOCVD-grown one. The broadening of the sharp ${ }^{4} \mathrm{I}_{15 / 2} \rightarrow{ }^{4} \mathrm{I}_{9 / 2} 4 f$ shell absorption peaks in the Er-implanted, HVPE-grown sample is apparently attributable to the fact that the concentration $\left(5.3 \times 10^{19}\right.$ ions $\left./ \mathrm{cm}^{3}\right)$ of the implanted $\mathrm{Er}^{3+}$ ions is larger than that $\left(2 \times 10^{18}\right.$ ions $/ \mathrm{cm}^{3}$ ) in the Er-implanted MOCVD-grown sample; a similar broadening of the sharp absorption peaks was observed in the PLE spectrum of MOCVD-grown GaN implanted with $10^{15} \mathrm{Er}$ ions $/ \mathrm{cm}^{2}$ at $350 \mathrm{keV}$ reported in Ref. 11 . While the sharpness of the PLE peaks in the PLE spectrum of the Er-implanted MOCVD-grown GaN indicates that isolated Er ions on Ga atomic positions are on identical, high-quality sites in this material [4], the spectral broadening of the direct $4 f$ PLE absorption peaks for the Er-implanted HVPE-grown sample implies moderate site-to-site variation in the crystal field.

The seven distinct optically active $\mathrm{Er}^{3+}$ centers observed previously in the Er-implanted MOCVD-grown GaN annealed at $900{ }^{\circ} \mathrm{C}$ (Ref. 3) have been labeled on the basis of their excitation wavelengths, as follows: above-gap (A), blue (B), orange (O), red (R), near-IR (N), $4 f$ 
(F) and violet (V). This labeling system has been used to identify the PLE absorption bands associated with the selective excitation of these $\mathrm{Er}^{3+}$ centers in the PLE spectra in Figs. $2 \mathrm{c}$ and $3 \mathrm{c}$. Figures 4, 5, and 6 show the "violet-pumped", "blue-pumped", and "red-pumped" PL spectra excited by 404, 458 and $633 \mathrm{~nm}$ light, respectively, that correspond with the similarly labeled PLE bands in Fig. 2c [4]. The "violet-pumped" PL spectra presented in Fig. 4 demonstrate that the PL peaks in the "violet-pumped" PL spectrum of the Er-implanted HVPE-grown GaN are the same as those in the Er-implanted MOCVD-grown GaN, while there was no $\mathrm{Er}^{3+}$ emission from the in situ Er-doped GaN when excited by $404 \mathrm{~nm}$ (violet) light. In Fig. 5, the broadened PL peaks in the "blue-pumped" PL spectrum of the in-situ Er-doped sample do not correspond to the B-labeled PL peaks associated with the "blue" $\mathrm{Er}^{3+}$ site observed in the Er-implanted MOCVD-grown sample, but the PL of the Er-implanted HVPE-grown sample includes both the B- and A-labeled PL peaks. The "red-pumped" PL spectra in Fig. 6 show that while the $633 \mathrm{~nm}$ light excites the $\mathrm{R}$ (red)-labeled $\mathrm{Er}^{3+}$ emission as well as the $\mathrm{B}-, \mathrm{A}-, \mathrm{N}-$, and O-labeled bands in the Er-implanted MOCVD GaN sample (Fig. 6b), only the B-labeled PL peaks are observable in the PL of the Er-implanted HVPE-grown sample (Fig. 6a), and no $\sim 1540 \mathrm{~nm}$ emission is excited from the in situ Er-doped GaN.

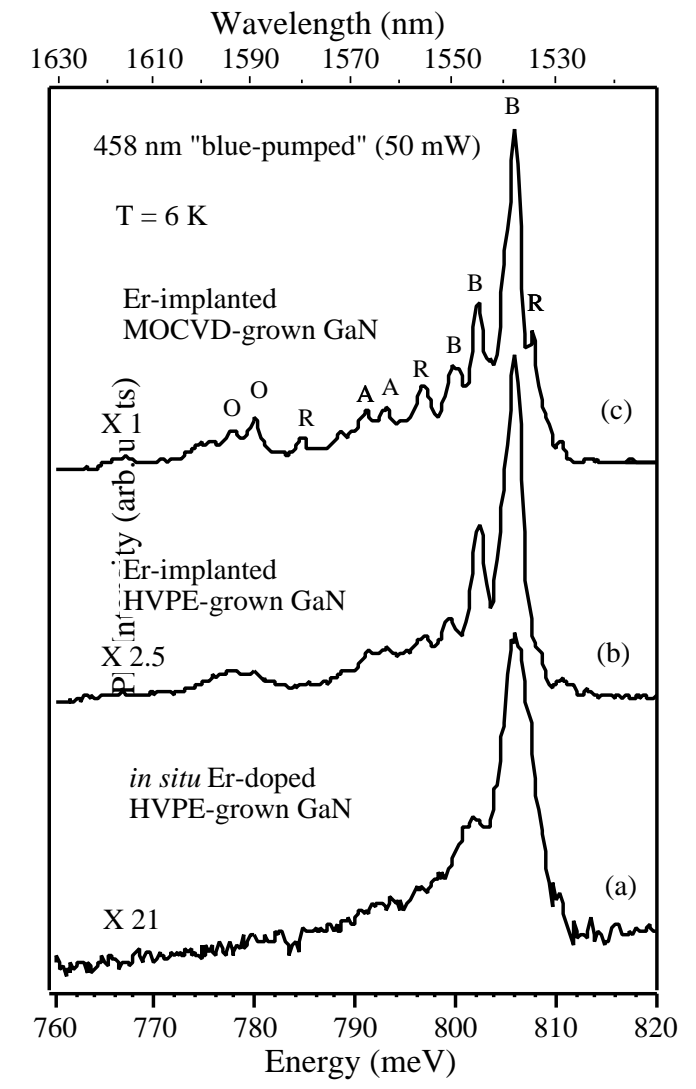

Fig. 5. The "blue-pumped" PL spectra as excited by blue light $(458 \mathrm{~nm})$.

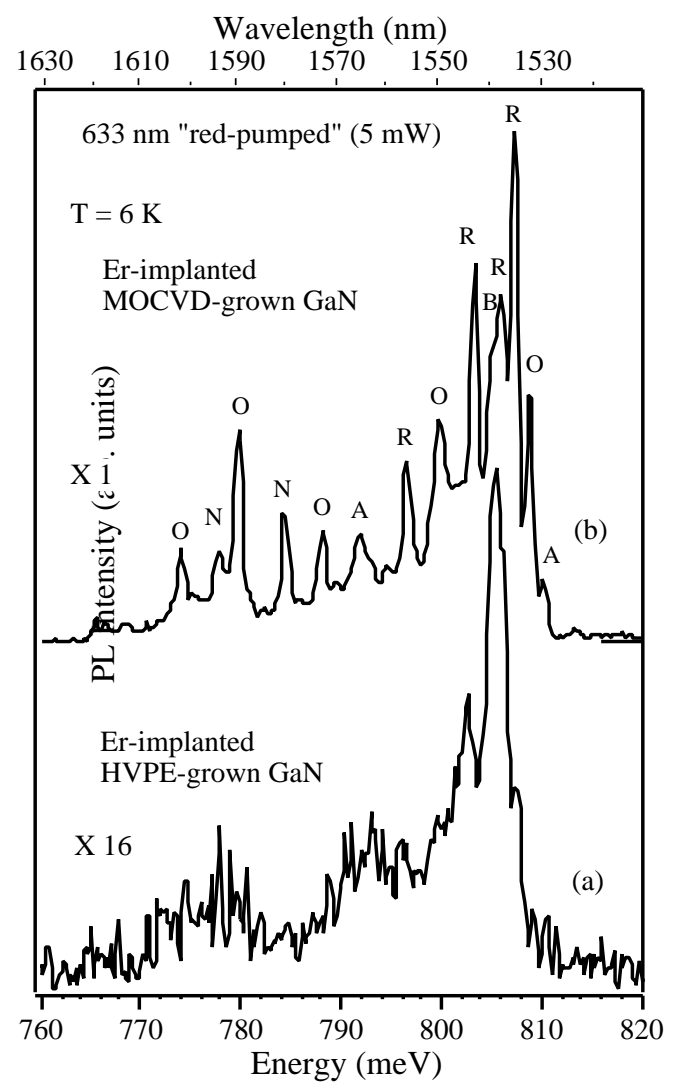

Fig. 6. The "red-pumped" PL spectra as excited by red light $(633 \mathrm{~nm})$.

The "4f-pumped" PL spectra in Fig. 7 were excited by $809 \mathrm{~nm}$ light that corresponds to direct $\mathrm{Er}^{3+} 4 f$-band absorption. Significantly, the " $4 f$-pumped" PL spectrum of the Er-implanted HVPE-grown GaN (Fig. 7b) shows sharply-structured PL peaks that are nearly identical in peak energy and relative intensities to those of the Er-implanted MOCVD-grown GaN PL spectrum of Fig. 7c. This indicates that most of the Er atoms in the Er-implanted HVPE-grown GaN occupy a 
single type of high-concentration, isolated $\mathrm{Er}^{3+}$ center, that can only be excited by direct $4 f$-band absorption, and that this is the same high-concentration $\mathrm{Er}^{3+}$ center previously observed by direct $4 f$-band pumping in Er-implanted MOCVD-grown GaN [3,4]. In contrast, $4 f$ pumping of the $\mathrm{Er}^{3+}$ dopants in the in-situ Er-doped GaN excites a broad $1540 \mathrm{~nm}$ PL spectrum (Fig. 7a) that is identical to the "green-pumped" PL spectrum (Fig. 1a) and the "blue-pumped" PL spectrum (Fig.

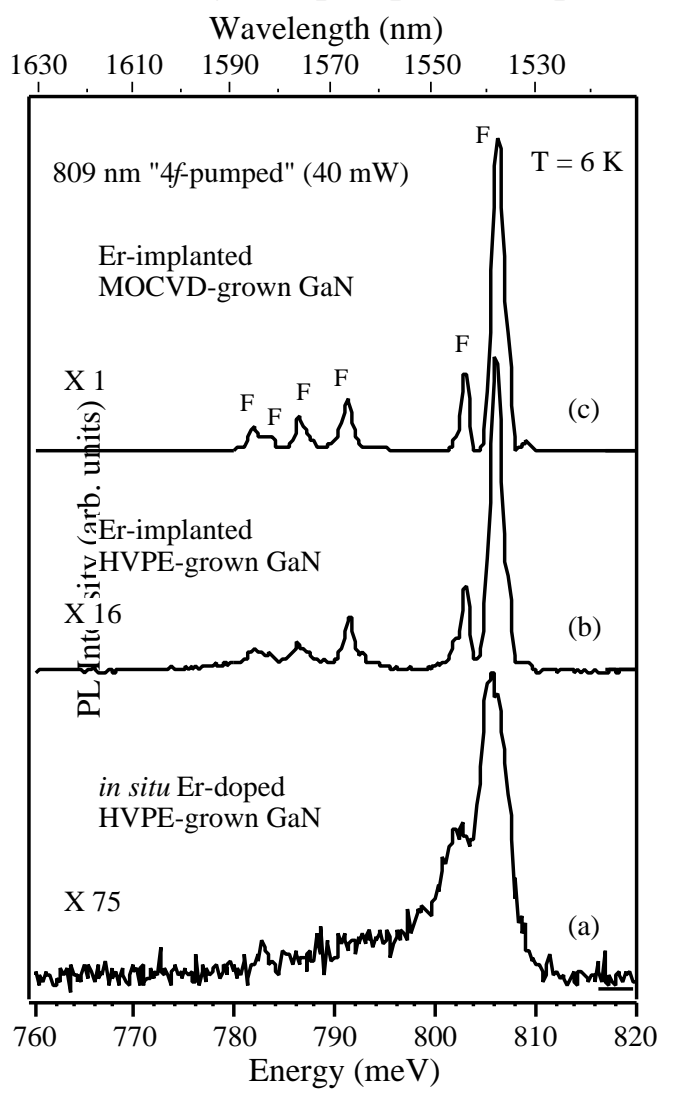

Fig. 7. The " $4 f$-pumped" PL spectra as excited by $809 \mathrm{~nm}$ light. 5a) for this sample, clearly demonstrating the absence in the in-situ-doped material of the multiple, selectively excited, discrete $\mathrm{Er}^{3+}$ sites or centers that are observed in the Er-implanted GaN. The broad $4 f$-pumped $\mathrm{Er}^{3+}$ PL spectrum confirms our earlier suggestion that the Er dopants in the insitu-doped sample occupy sites located in disordered regions of the material and therefore experience site-to-site variations in the crystal field.

\section{CONCLUSIONS}

Site-selective PLE and PL spectroscopy have been carried out at $6 \mathrm{~K}$ on the $1540 \mathrm{~nm}^{4} \mathrm{I}_{13 / 2}$ $\rightarrow{ }^{4} \mathrm{I}_{15 / 2}$ emission of $\mathrm{Er}^{3+}$ in in situ Er-doped and Er-implanted GaN grown by HVPE. The PLE and PL spectra of these two different Er-doped HVPE-grown GaN films are compared in this study with Er-implanted GaN grown by MOCVD to see if the multiple, selectively excited, discrete $\mathrm{Er}^{3+}$ sites or centers observed in our previous studies of Er-implanted $\mathrm{GaN}$ are present in Er-doped GaN synthesized by other growth, doping and annealing procedures.

The PLE and PL spectroscopy of this study reveal that four of the seven different $\mathrm{Er}^{3+}$ sites observed in Er-implanted MOCVD-grown GaN annealed at $900{ }^{\circ} \mathrm{C}$ under a flow of $\mathrm{N}_{2}$ are present in Er-implanted HVPE-grown GaN annealed at $800{ }^{\circ} \mathrm{C}$ in an $\mathrm{NH}_{3} / \mathrm{H}_{2}$ atmosphere. In contrast, the in situ Er-doped HVPE-grown GaN exhibits a single, broad $\sim 1540 \mathrm{~nm} \mathrm{Er}^{3+} \mathrm{PL}$ spectrum whose lineshape is independent of excitation wavelength. This broad $\mathrm{Er}^{3+} \mathrm{PL}$ spectrum, which is reminiscent of emission from an Er-doped glass, prompts the suggestion that the Er dopants in the in-situ-doped sample occupy sites located in disordered regions of the material characterized by site-to-site variations in the crystal field.

\section{ACKNOWLEDGMENTS}

This work was supported by NSF under the Engineering Research Centers Program (ECD 89-43166), DARPA (MDA972-94-1-004), and the JSEP (0014-90-J-1270).

\section{REFERENCES}


1. S. Kim, S. J. Rhee, D. A. Turnbull, E. E. Reuter, X. Li, J. J. Coleman, and S. G. Bishop, Appl. Phys. Lett. 71, 231 (1997).

2. S. Kim, S. J. Rhee, X. Li, J. J. Coleman, and S. G. Bishop, Appl. Phys. Lett. 71, 2662 (1997).

3. S. Kim, S. J. Rhee, X. Li, J. J. Coleman, and S. G. Bishop, Mater. Res. Soc. Symp. Proc. 501, to be published (1998).

4. S. Kim, S. J. Rhee, X. Li, J. J. Coleman, and S. G. Bishop, J. Electron. Mater., 27, 246 (1998).

5. M. Thaik, U. Hommerich, R. N. Schwartz, R. G. Wilson, and J. M. Zavada, Appl. Phys. Lett. 70, 2641 (1997).

6. X. Wu, U. Hommerich, J. D. MacKenzie, C. R. Abernathy, S. J. Pearton, R. N. Schwartz, R. G. Wilson, and J. M. Zavada, Appl. Phys. Lett. 70, 2126 (1996).

7. D. M. Hansen, R. Zhang, N. R. Perkins, S. Safvi, L. Zhang, K. L. Bray and T. F. Kuech, Appl. Phys. Lett. 72, 1244 (1998).

8. D. A. Turnbull and S. G. Bishop, J. Non-Cryst. Solids., 223, 105 (1998).

9. N. F. Mott and E. A. Davis, Electronic Processes in Non-Crystalline Materials, (Clarendon Press, Oxford, 1979), p. 273.

10. F. Gan, Laser Materials, (World Scientific, New Jersey, 1995), p. 220.

11. J. T. Torvik. C. H. Qui, R. J. Feuerstein, J. I. Pankove, F. Namavar, J. Appl. Phys. 81, 6343 (1997). 